\title{
TRMM Mapping using Different Models in Iraq
}

\author{
Hussain Zaydan Ali \\ Ministry of Science and \\ Technology \\ Baghdad/Iraq
}

\author{
Salih M. Al-Qaraawi \\ Computer Engineering Dept. \\ University of Technology \\ Baghdad/Iraq
}

\author{
Ghusoon Eidan Arab \\ Computer Engineering Dept. \\ University of Technology \\ Baghdad/Iraq
}

\begin{abstract}
All the necessary water for life on earth originates from rain. For this reason, it is important to understand the spatial and temporal patterns of rainfall and their variability in order to gain knowledge about the balance of water dynamics for water resources management, and to plan strategies for solving many problems such as predicting natural hazards caused by heavy rain. The principal objectives of this paper are to validate an optimum interpolation method for the spatial analysis of monthly precipitation in Iraq. The rootmean-square error (RMSE) of the verification stations (the error of the predicted value at the station location from the observed value at the station). The mean error (ME) was used to detect any bias in the estimates. In this paper, we used the kriging interpolation to estimate the rainfall distribution in Iraq. Geostatistical interpolation techniques were implemented in a Geographic Information System (GIS) to study the spatial variability of monthly TRMM in Iraq using different models (Spherical, Exponential and Gaussian).
\end{abstract}

\section{Keywords}

GIS, Kriging, Spherical Model, TRMM Interpolation, Iraq.

\section{INTRODUCTION}

Prediction of spatial attributes has attracted significant research interest in recent years. It is challenging especially when spatial data contain errors and missing values. Geostatistical estimators are used to predict the missing attribute values from the observed values of known surrounding data points, a general form of which is referred as kriging in the field of geographic information system and remote sensing. A large sampling effort is required to produce an accurate geostatistical maps and the extraction and analysis of each sample is often expensive, the effectiveness of a particular sampling scheme is dependent upon the spatial variability of the quantity being measured .In this paper, we used the kriging methods to estimate the rainfall distribution in Iraq. Literatures suggest that there is no single preferred method of interpolation, and the selection of interpolation method is usually based on the available data, desired level of accuracy, and available resources. Kriging is an optimal and unbiased linear technique for estimation. Compared with the traditional interpolation methods, it provides the 'best' possible estimates of unknown values from sample data [1]. Kriging interpolation method, used here, is one of the most popular and useful methods to predict unknown values from data observed at known locations, especially in geo-statistical. This method produces visually appealing map from irregularly space data and uses variogram to express trends suggested in the data [2]. Geostatistical Analyst derives a surface using the values from the measured locations to predict values for each location in the landscape. Geostatistical Analyst provides two groups of interpolation techniques: deterministic and geostatistical. All methods rely on the similarity of nearby sample points to create the surface. Deterministic techniques use mathematical functions for interpolation. Geostatistics relies on both statistical and mathematical methods, which can be used to create surfaces and assess the uncertainty of the predictions. Geostatistical Analyst, in addition to providing various interpolation techniques, also provides many supporting tools. These tools allow you to explore and gain a better understanding of the data so that you create the best surfaces based on the available information [3]. Kriging methods that produce prediction maps are listed below:

-Ordinary Kriging (OK).

-Simple Kriging (SK).

-Universal Kriging (UK).

-Disjunctive Kriging (DK).

\section{DATA SOURCE}

The data source used in this study was acquired by the Tropical Rainfall Measuring Mission (TRMM). The data were processed by the TRMM Science Data and Information System (TSDIS) archived and distributed by the Goddard Distributed Active Archive Center. TRMM is an international project jointly sponsored by the Japan Aerospace Exploration Agency (JAXA, previously known as National Space Development Agency or NASDA) and the U.S. National Aeronautics and Space Administration (NASA) Office of Earth Sciences. The data used in this study were processed using the GESDISC Interactive Online Visualization and analysis Infrastructure (Giovanni) as part of the NASA's Goddard Earth Sciences (GES) Data and Information Services Center (DISC) [4].

\section{MATHEMATICAL MODEL IMPLEMENTATION}

The main models are Spherical model, Exponential model and Gaussian model. The actual process of fitting a model to an empirical semivariogram is much more of an art than a science. The selected model influences the prediction of unknown values, it represents the true spatial distribution of the variable.

$\gamma(h)=\left\{\begin{array}{l}C_{0}+C_{1}\left[\frac{3 h}{2 a}-\frac{1}{2}\left(\frac{h}{a}\right)^{2}\right] h \leq a \\ C_{0}+C_{1} h \geq a\end{array}\right\} \quad$ Spherical Model (1) 
$\gamma(h)=C_{0}+C_{1}\left[1-\exp \left(\frac{h^{2}}{a^{2}}\right)\right]$ Exponential Model (2)
$\gamma(h)=C_{0}+C_{1}\left[1-\exp \left(\frac{h}{a}\right)\right]$ Gaussain Model (3)

Where $\gamma(h)$ is semivarogram, $C_{0}$ is the nugget value, (h) is lag distance, and (a) is the range. The spherical model actually reaches the specified sill value $\mathrm{C}$, at the specified range (a) [5]. While the exponential and Gaussian approach the sill asymptotically, with a representing practical range; the distance at which the semivariance reaches $95 \%$ of the sill value see fig.1.

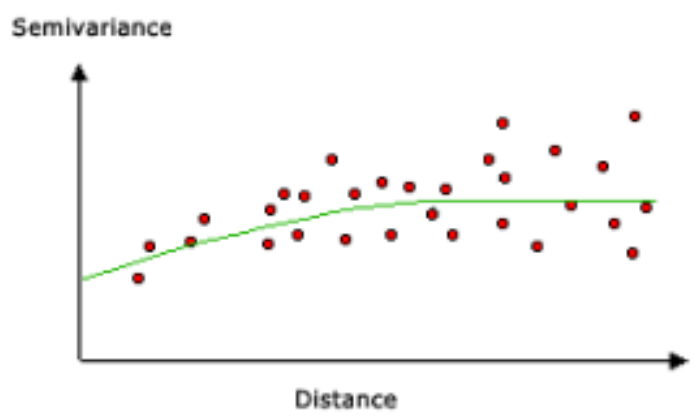

Fig.1: Spherical Model

\section{RESULTS AND DISCUSSION}

In this paper, the semi-variogram was modeled as Spherical, Gaussian and Exponential type function. Cross validation criteria used to assess the best model. Cross validation allows determining how good model is. The goal should be have standardized mean prediction errors near to 0 , small root mean square prediction errors, average standard error near root mean square prediction errors, and standardized root mean square prediction errors near to 1 [6].

\subsection{CASE STUDY 1: Comparison of Surface Models for March 2003}

It is common practice to create many surfaces before one is identified as "best" and will be final in it. In fig.2, fig.3 and fig. 4 using cross validation criteria to comparing Exponential model, Gaussian model and Spherical model based TRMM data for March 2003, it notice the spherical model is the best from others because minimum mean predicted error.

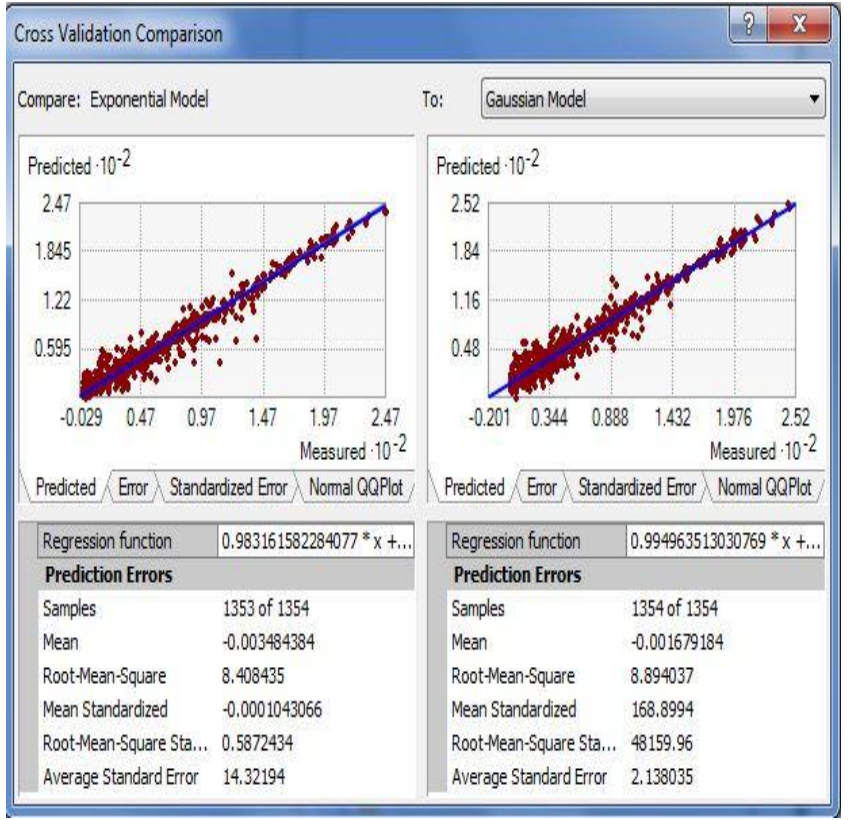

Fig.2: Comparison of Exponential Model and Gaussian Model for case study 1

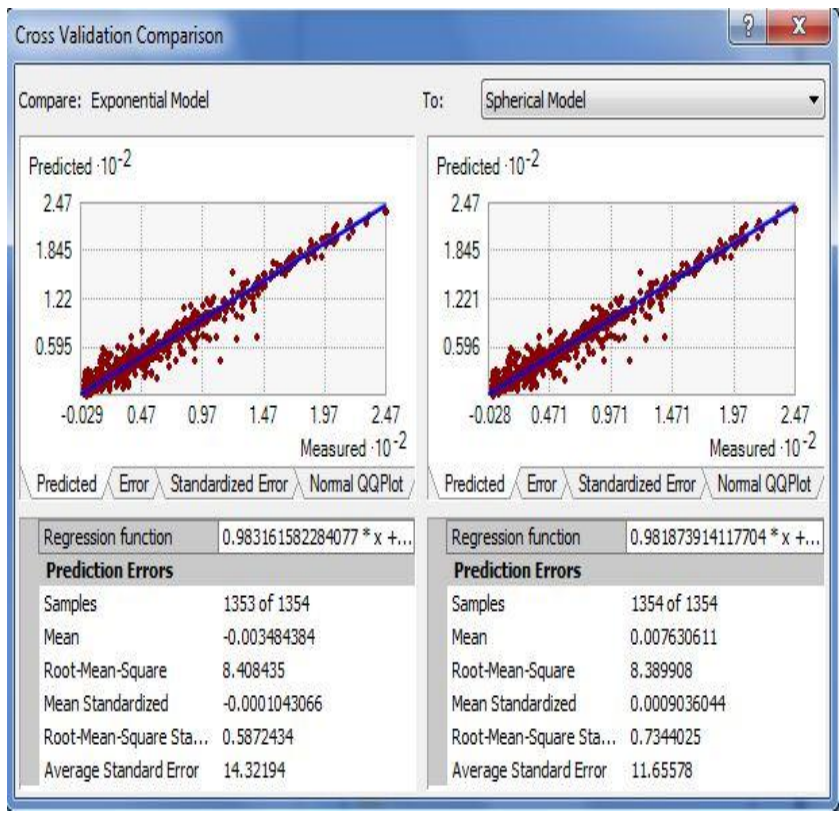

Fig.3: Comparison of Exponential Model and Spherical Model for case study 1 


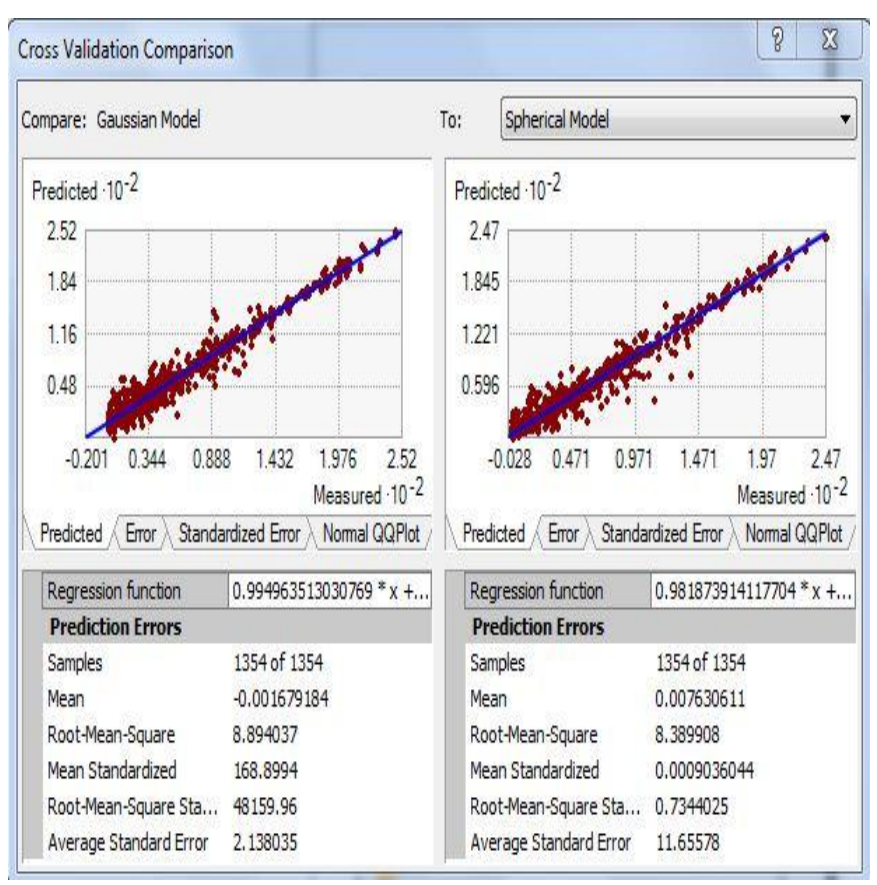

Fig.4: Comparison of Gaussian Model and Spherical Model for case study 1

\subsection{CASE STUDY 2: Comparison of Surface Models for November 2009}

In fig.5, fig.6 and fig.7 using cross validation criteria to comparing Exponential model, Gaussian model and Spherical model based TRMM data for November 2009, it notice the spherical model is the best from others because minimum mean predicted error.

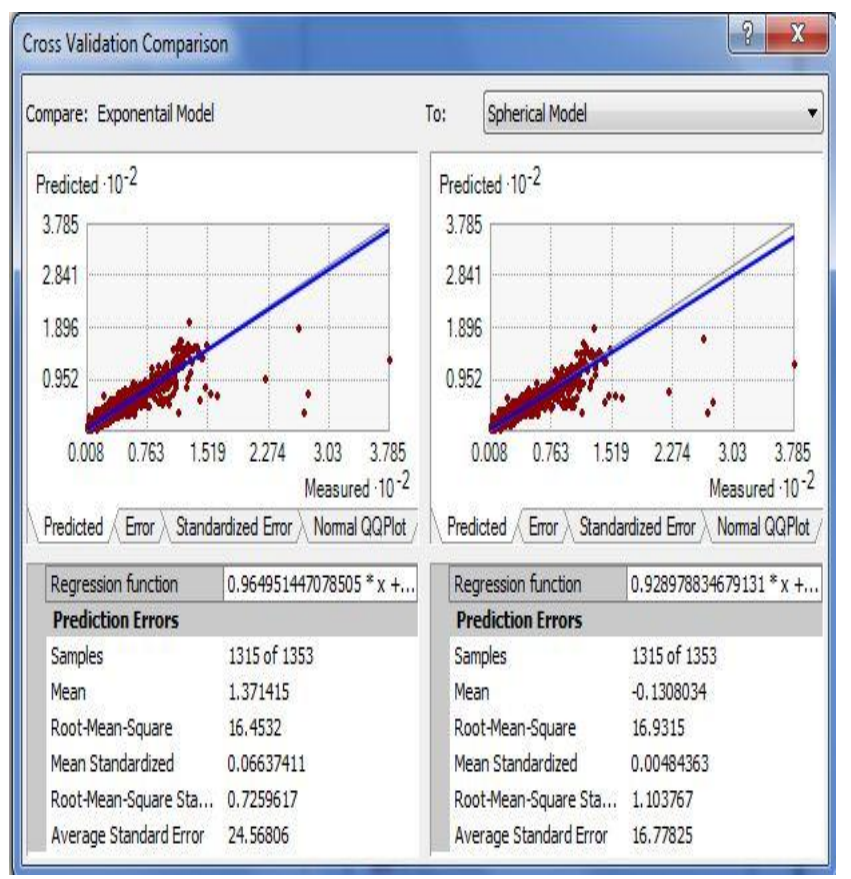

Fig.5: Comparison of Exponential Model and Spherical Model for case study 2

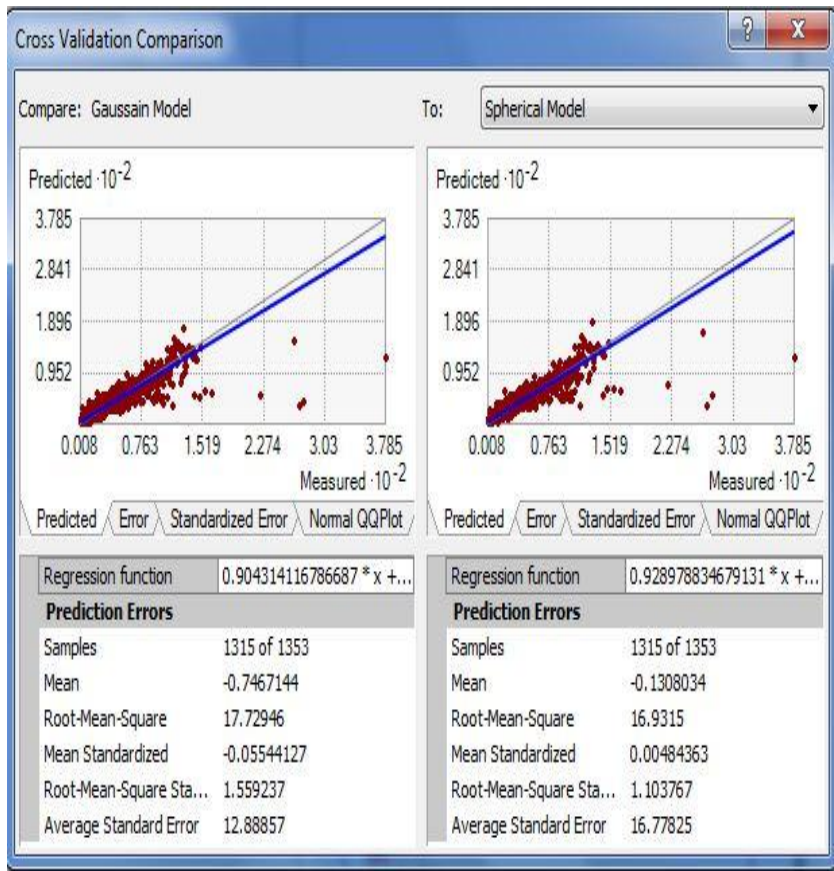

Fig.6: Comparison of Gaussian Model and Spherical Model for case study 2

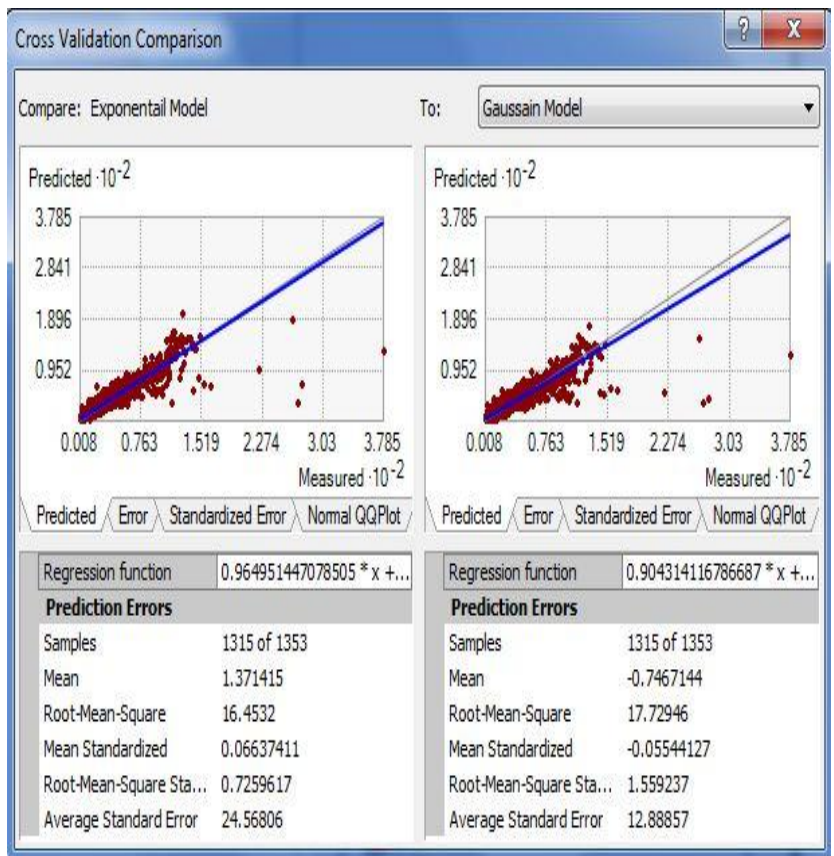

Fig.7: Comparison of Exponential Model and Spherical Model for case study 2

\subsection{CASE STUDY 3: Comparison of Surface Models for January 2013}

In fig.8, fig.9 and fig.10 using cross validation criteria to comparing Exponential model, Gaussian model and Spherical model based TRMM data for January 2013 , it notice the spherical model is the best from others because minimum mean predicted error. 


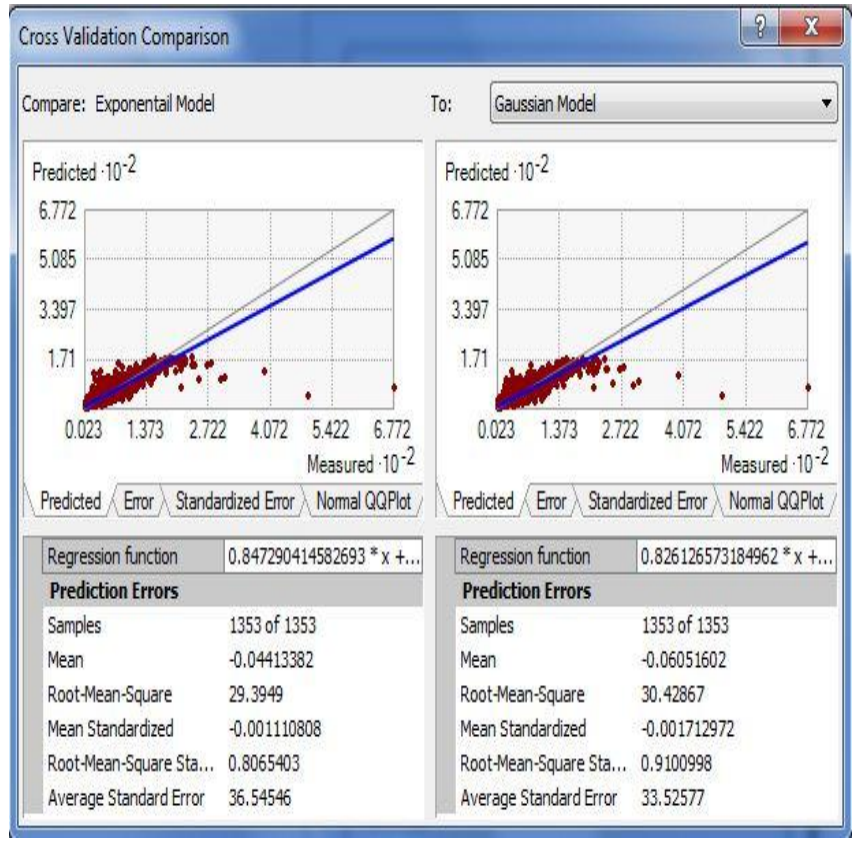

Fig.8: Comparison of Exponential Model and Gaussian Model for case study 3

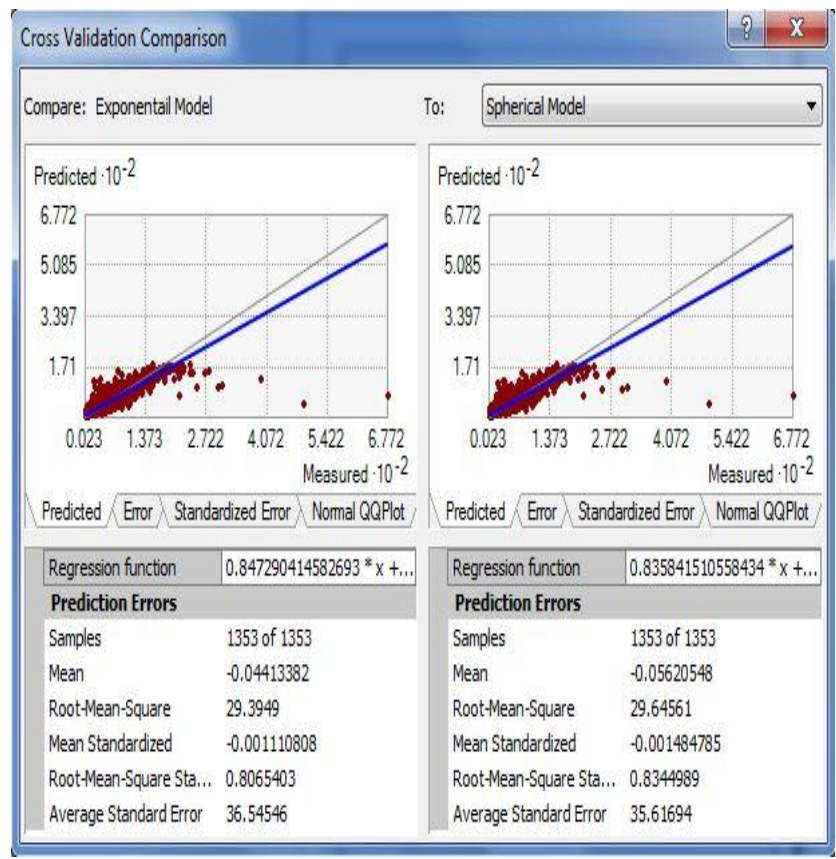

Fig.9: Comparison of Exponential Model and Spherical Model for case study 3

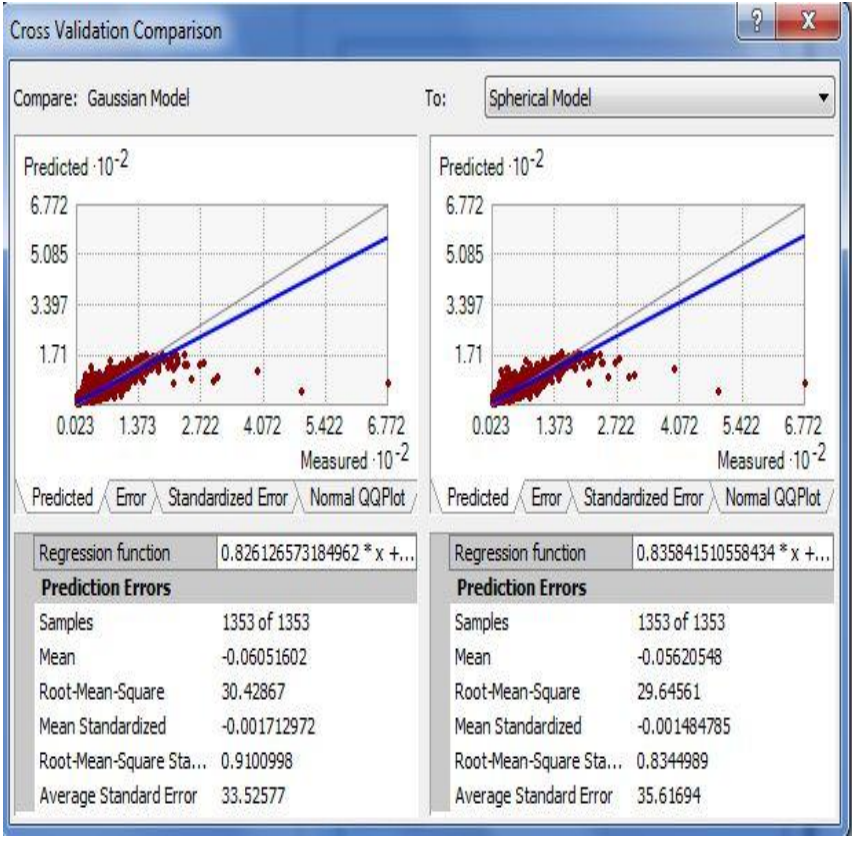

Fig.10: Comparison of Gaussian Model and Spherical Model for case study 3

See fig.11, fig.12 and fig.13 presented prediction mapping for TRMM data. The spherical model is the best used models based on cross validation criteria. Rainfall maps generated by the spherical model show the ability to capture precipitation structure in Iraq.

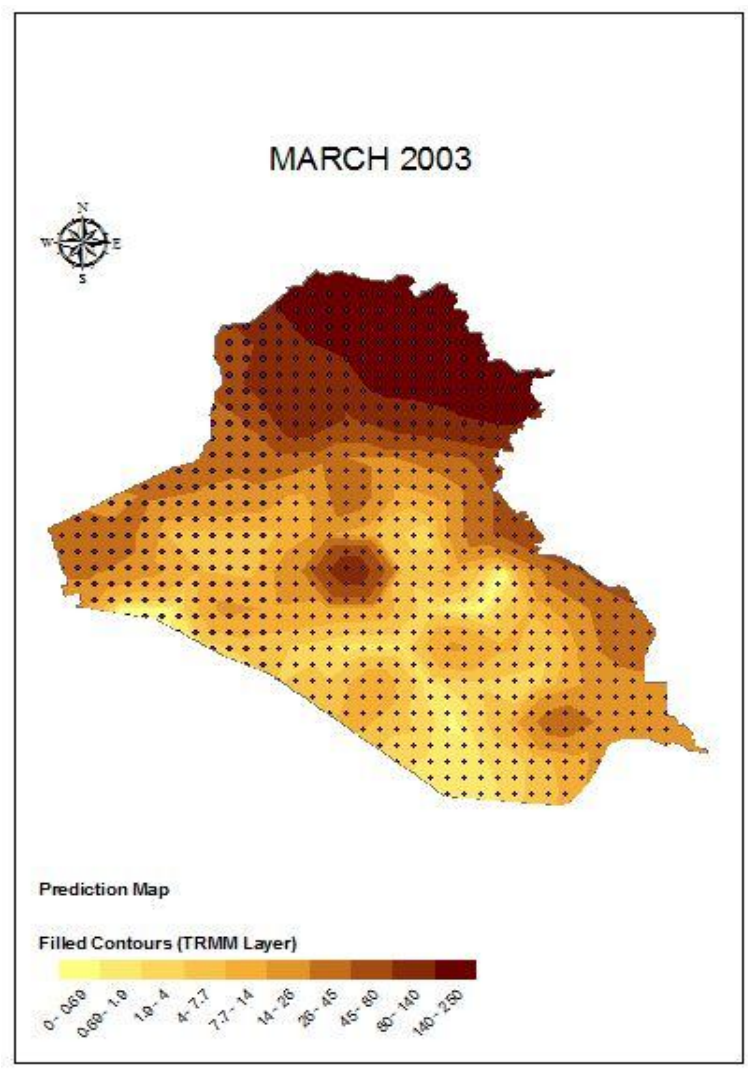

Fig.11: TRMM mapping for March 2003 


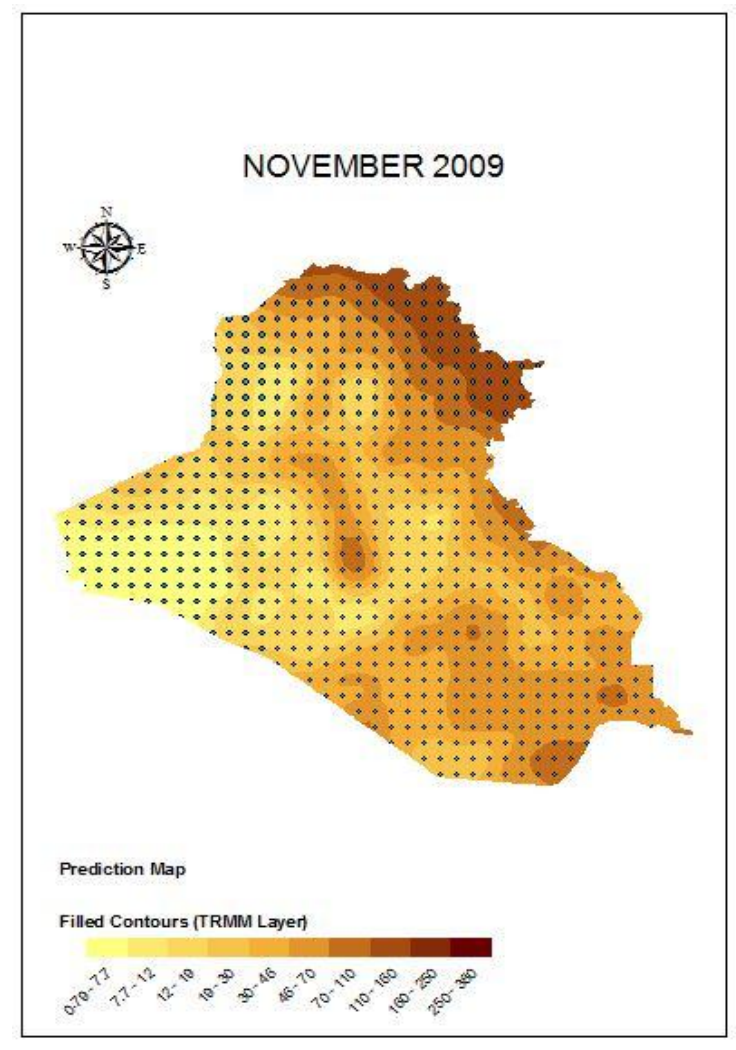

Fig.12: TRMM mapping for November 2009

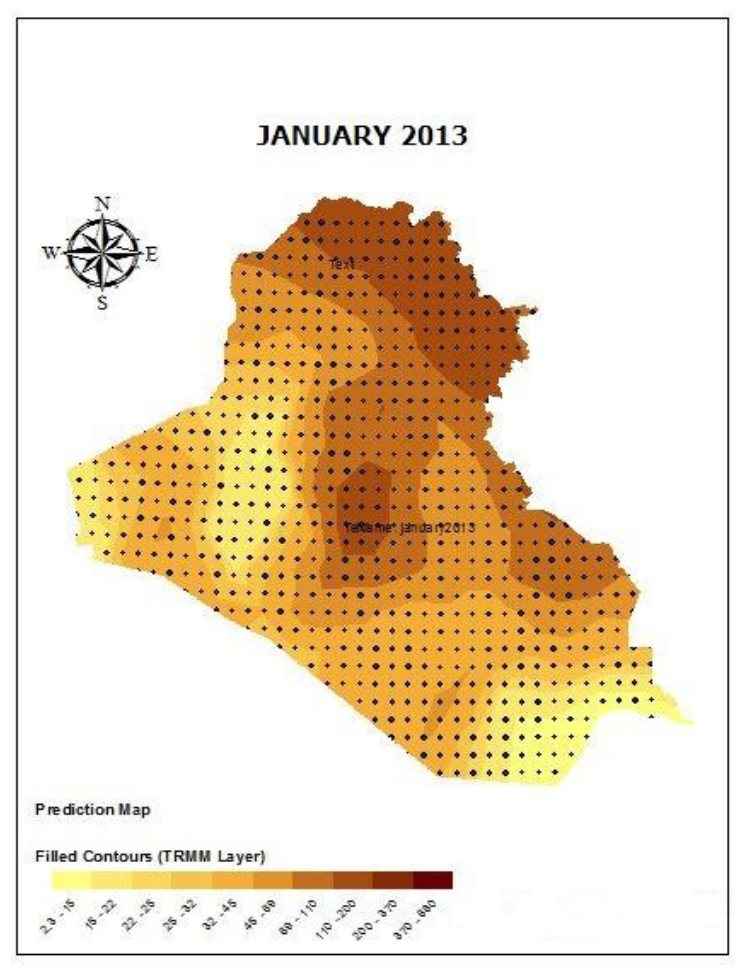

Fig.13: TRMM mapping for January 2013
From Fig.11,Fig.12 and Fig.13 can be concluded TRMM data is more accuracy than weather station data because of TRMM data give more than 679 points per Iraq while weather stations give small number of points per Iraq.

\section{CONCLUSION}

This paper has showed that the Spherical model with TRMM data has a noticeable performance advantage compared to the Gaussian model and Exponential model with TRMM data in terms environmental models. The root-mean-square error (RMSE) of the verification and the mean error (ME) were used to detect any bias in the estimates. Kriging methods gave encouraging results when applied to the data rains in Iraq and achieved high precision of the results predicted. Rainfall maps generated by the spherical model show the ability to capture precipitation structure in Iraq.

\section{REFERENCES}

[1] Y. Feng, X. Lei, and Y. Lu, 2004, "Interpretation of Pixel-missing Patch of Remote Sensing Image with Kriging Interpolation of Spatial Statistics," Journal of Remote Sensing, vol. 8(4), pp. 317-322.

[2] Jinyan Zhan, Nana Shi, Xiangzheng Deng, Hongbo Su, Dongsheng Qiu, 2011, "Interpolating The information of Site-Based Soil Organic Carbon Stocks Into Surface: A Case Study in The North China Plain",Transaction in IEEE, 978-1-4244-2808.

[3] Environment System Research Institute documentation, 2003, "Using ArcGIS Geostatistical Analyst", USA.

[4] Hussain Ali, Salih M.Al-Qaraawi, Ghusoon Arab, 2014,"Optimal Interactive Rainfall Maps (Iraq) in Client Side and Google Earth", International Journal of Computers and Technology, vol.13, no.1.

[5] Ragab Elsayed Rabeiy,2010,"Spatial Modeling of Heavy Metal Pollution of Forest Soils in a Historical Mining Area Using Geostatistical Methods and Air Dispersion Modeling", Doctoral Thesis, Clausthal University of Technology ,Germany.

[6] Environment System Research Institute, 2008, help documentation of Geostatistical Analyst, USA. 